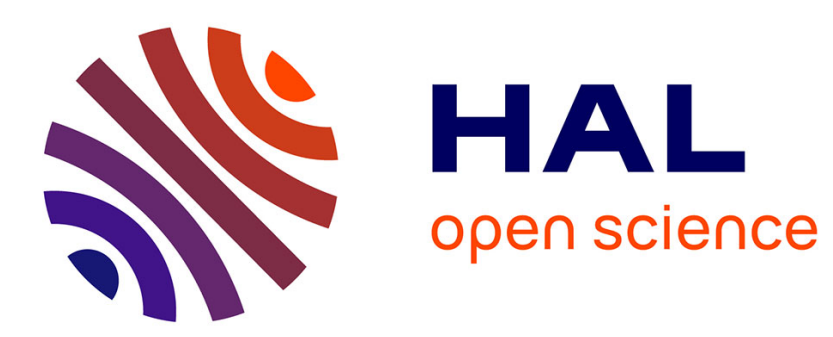

\title{
A Fuzzy Approach for the Kinematic Reliability Assessment of Robotic Manipulators
}

Fabian A Lara-Molina, Didier Dumur

\section{To cite this version:}

Fabian A Lara-Molina, Didier Dumur. A Fuzzy Approach for the Kinematic Reliability Assessment of Robotic Manipulators. Robotica, inPress, 10.1017/S0263574721000187 . hal-03167274

\section{HAL Id: hal-03167274 \\ https://hal.science/hal-03167274}

Submitted on 16 Mar 2021

HAL is a multi-disciplinary open access archive for the deposit and dissemination of scientific research documents, whether they are published or not. The documents may come from teaching and research institutions in France or abroad, or from public or private research centers.
L'archive ouverte pluridisciplinaire HAL, est destinée au dépôt et à la diffusion de documents scientifiques de niveau recherche, publiés ou non, émanant des établissements d'enseignement et de recherche français ou étrangers, des laboratoires publics ou privés. 


\title{
A Fuzzy Approach for the Kinematic Reliability Assessment of Robotic Manipulators
}

\author{
Fabian A. Lara-Molina $\dagger^{*}$ and Didier Dumur $\ddagger$ \\ $\dagger$ †ederal University of Technology - Paraná, Cornélio Procópio, PR 86300-00 Brazil \\ $\ddagger$ Laboratoire des Signaux et Systémes, CentraleSupélec-CNRS-Univ. Paris-Sud, Univ. \\ Paris-Saclay, Control Department, 91 192, Gif sur Yvette cedex, France
}

(Accepted MONTH DAY, YEAR. First published online: MONTH DAY, YEAR)

\section{SUMMARY}

This paper aims at developing a novel method to assess the kinematic reliability of robotic manipulators based on the fuzzy theory. The kinematic reliability quantifies the probability of obtaining positioning errors within acceptable limits. For this purpose, the fuzzy reliability evaluates the effect of the joint clearances on the end effector position to compute a failure possibility index. As an alternative to the conventional methods reported in the literature, this failure possibility index conveys a novel assessment of the kinematic performance. The numerical results are compared with the well known probabilistic approach based on the Monte Carlo simulation (MCS).

KEYWORDS: kinematics; Manipulator; Fuzzy; Uncertainties; Clearances.

\section{Introduction}

The design criteria aims at quantifying the mechanical properties of the robotic manipulators. Several design criteria have been used to examine the kinematic and dynamic capabilities [1]. The definition of these design criteria is challenging due to the uncertainties associated to the operation of robotic manipulators [2]. The robotic manipulators are unavoidably affected by uncertainties in their geometric parameters produced by manufacturing and assembly error of the links, backlash positioning error

\footnotetext{
* Corresponding author. E-mail: fabianmolina@utfpr.edu.br
} 
of the actuators, and joints clearances. The calibration of the mechanism reduces the effect of manufacturing and assembly errors of the links significantly. However, the errors produced by joint clearances can not be correctly compensated by the calibration methods [3]. Moreover, joint clearances are necessary for the relative motion between the links; therefore, they are the most important source of error that affects the accuracy and repeatability of the mechanisms [4]. For this reason, it is necessary to develop computational methods to analyze the effects of joint clearances and uncertainties in the pose error of mechanisms.

The joint clearances in passive joints of parallel mechanisms produce unconstrained end effector motions when the active joints are blocked [5]. The clearances in the axisymmetrical joints were previously modeled by [6]. Moreover, the uncertainty effect of joint clearance has widely been studied by using probabilistic approaches [7-9]. Additionally, several approaches have been developed to assess the influence of joint clearances on the kinematic accuracy of mechanisms. The pose error of the links has been determined by using a kinematic method [10]. Moreover, the kinematic accuracy of parallel manipulators with joint clearances has been analyzed. A systematic analysis of the error of parallel manipulators was developed based on [10] by using standard convex optimization [11].

The kinetostatic reliability has been widely evaluated by using probabilistic approaches to evaluate the effect of random uncertainties. Consequently, several performance measures based on the probabilistic methods have been proposed to evaluate the kinematic reliability. The probability of failure defines the probability that the actual position of an end effector falls into a specified error limit $[8,16]$. The time-dependent reliability to assess the time-dependency effect of the motion error [7]. The kinematic sensitivity [12] finds out the influence of each error source on the kinematic reliability. And, the error sensitively indexes that reflect the mapping relationship to evaluate the level of kinematic accuracy under various configurations [14]. Alternative performance indexes have been proposed based on the sensitivity of the clearances [11]. Nevertheless, the fuzzy approach to evaluate uncertainties has not been used to assess the reliability of the robotic manipulator. As an alternative to the probabilistic methods, the fuzzy theory permits to model the uncertainty and to describe inaccurate information [17]. Consequently, the uncertainties can be modeled using fuzzy sets theory for applications in which the stochastic process that models the random uncertainties is unknown. Based 
on the fuzzy approach, a new index is formulated to evaluate the possibility that the positioning error exceeds an acceptable limit; the kinematic possibility is assessed by considering the maximum error, differently of the probability of failure that is related to how likely the error exceeds the acceptable limit. The fuzzy theory has successfully applied to control of robotic manipulators [18-20].

The present contribution proposes a novel method to assess the kinematic reliability of robotic manipulator based on the fuzzy theory. First, the clearance model with uncertain parameters is presented based on the axisymmetric joint model with uncertain parameters modeled as fuzzy variables. Then, the error propagation method determines the uncertain fuzzy pose error of the end effector produced by the joint clearances considering the kinematic constraints serial and parallel mechanism. Finally, the kinematic reliability that expresses the possibility that error exeeds a determined limit is computed. The novelty of the proposed method consists of estimating the possibility of kinematic failure associated with the maximum error produced by clearances; this approach permits defining the uncertainties for applications in which the probability density is unknown. Existing studies demand the knowledge of uncertain parameters probability densities.

This paper is organized into five sections. Initially, section 2 presents the literature review. Section 3 presents the methods that consider the axisymmetric model of joint clearances with the uncertainties, the error propagation method, and the fuzzy reliability approach. Next, section 4 presents the numerical results that encompass the assessment of the fuzzy reliability of a 3R serial manipulator and a Cartesian parallel manipulator. Finally, the conclusion is presented.

The following notations have been used in this paper: 


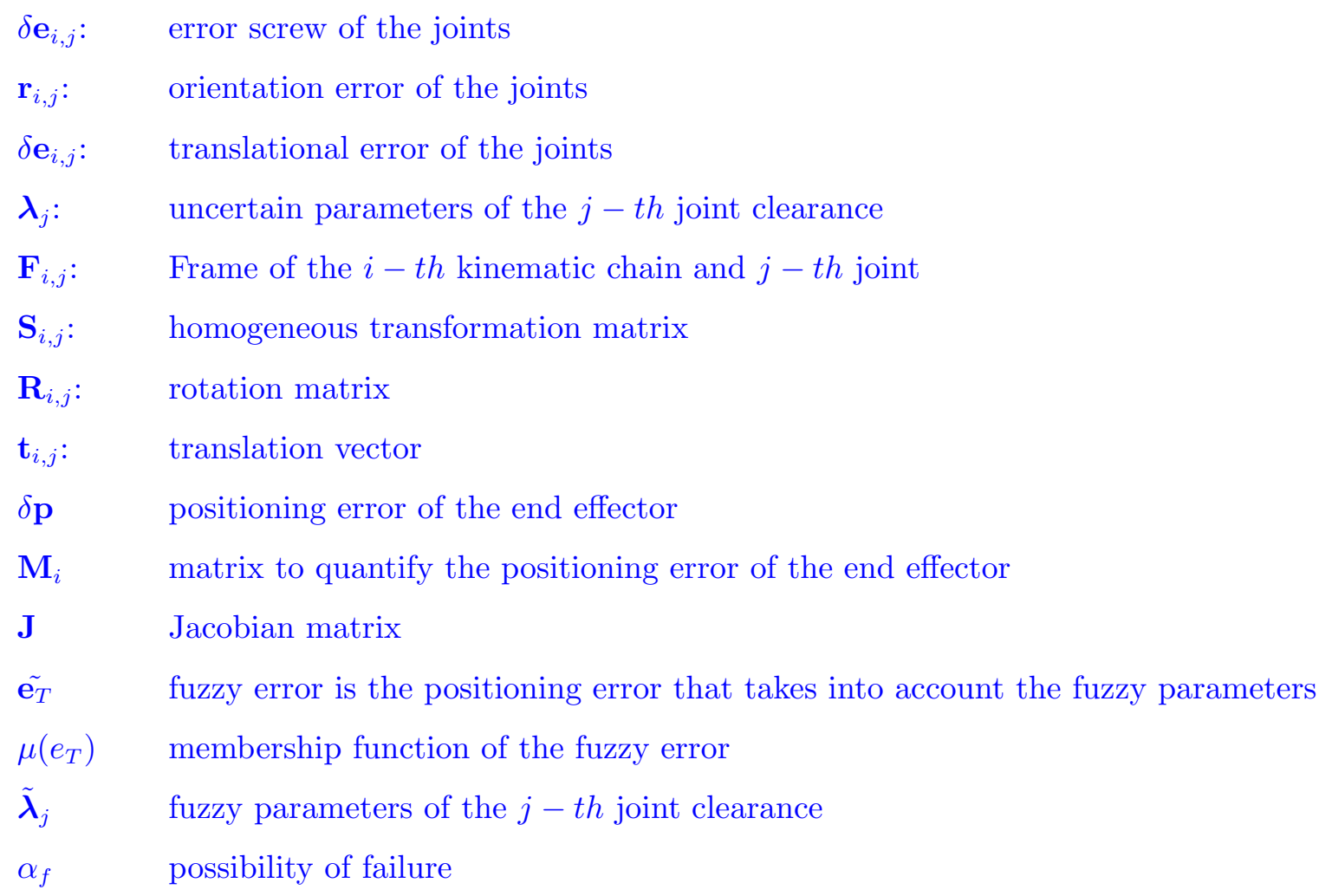

\section{Literature Review}

This section aims at presenting the prior work on the kinematic reliability of robotic manipulators and mechanisms. This review is mainly focused on the methods that have been developed in order to illustrate the novelty of the proposed approach based on the fuzzy kinematic reliability of manipulators.

The probabilistic approach has widely used in the literature to evaluate the reliability of robotic manipulators. The kinematic reliability aims at estimating the probability of failure produced by the unavoidable effect of uncertainties; the effect of the clearances on the kinematic accuracy has taken the attention of researchers regarding the kinematic performance $[7,8,24]$. Moreover, the kinematic reliability has recently emerged as an alternative criterion to evaluate the effects of the uncertainties in manipulators [12]. Several methods have been established to evaluate the kinematic reliability. Kim et al (2010) evaluated the kinematic reliability of manipulators using the advanced first order second moment (AFOSM) method. Pandey and Zang (2012) used the fractional moments to efficiently compute the kinematic reliability such that the position error remains within acceptable limits. Cui et al. (2015) computed the kinematic reliability using the Monte Carlo simulation and they evaluated three error sensitivity criteria based on the singular 
value decomposition of the error translation matrix. Zhan et al. (2018) proposed a hybrid method based on the first order second moment to evaluate the uncertainties of a planar parallel manipulator modeled as random and interval variables. Xu (2018) studied the influence of each error source on the kinematic reliability of a delta parallel manipulator. Zhang and Han (2020) developed an efficient reliability analysis method to account for random dimensions and joint angles of robotic mechanisms.

As an alternative to the probabilistic methods, a non-probabilistic approach based on the Lagrange multipliers and Taylor expansion has been proposed [23] to evaluate the kinematic reliability of the mechanism with clearances. Moreover, a time-dependent reliability index was described based on a combination of the nonprobabilistic interval process and first-passage theories. [25].

The methods based on a probabilistic approach computes an indirect measure of the effect of uncertainties based on the probability of failure. The probability of failure expresses how likely the positioning error is greater than a maximum limit; it depends on the probability distribution imposed on the uncertain parameters. Nevertheless, in several applications, it is not possible to find the probability distribution of the uncertain parameters based on experimental measures [23]. Additionally, it could be useful to define the kinematic reliability based on the positioning error rather than using indirect indexes based on the probability of failure. Therefore, the present manuscript proposes a fuzzy approach to evaluate the kinematic reliability of robotic manipulators with clearances that simplified the modeling of uncertainties and derive a new index based on the maximum positioning error.

\section{Methods}

This section presents the method used to compute the fuzzy kinematic reliability. Initially, the definition of joint clearance is presented as a function of the uncertain parameters. Based on the joint clearances model, a method is defined to obtain the total positioning error produced by the joint clearances on the end effector of the serial manipulators and parallel manipulators. Finally, the approach to compute the fuzzy kinematic reliability is presented based on the positioning error method. 


\subsection{Joint Clearance Model}

The axisymmetric model of the joint clearance was inspired in the model presented by [6] and [11]. The novel developments of the contribution consist of introducing the fuzzy uncertainties within the parameters that define the clearances.

Clearances introduce additional and uncontrollable degrees of freedom within the joints according to the axisymmetric joint clearance model that considers the joint axis along the $z$-axis (see Fig. 1). These additional degrees of freedom can be either rotational and translational; consequently, the pose error at the local frame of the joint can be modeled using the error screw $\delta \mathbf{e}_{i, j}$, thus:

$$
\delta \mathbf{e}_{i, j}=\left[\delta \mathbf{r}_{i, j} \delta \mathbf{t}_{i, j}\right]^{T}
$$

where $i$ is the index of the kinematic chain, and $j$ is the index of the joint in the respective $i^{\text {th }}$ kinematic chain, $\delta \mathbf{r}_{i, j}=\left[\delta r_{i, j, x} \delta r_{i, j, y} \delta r_{i, j, z}\right]^{T}$ is the orientation error, and $\delta \mathbf{t}_{i, j}=$ $\left[\delta t_{i, j, x} \delta t_{i, j, y} \delta t_{i, j, z}\right]^{T}$ is the translational error produced by the clearances with respect to the local frame $F_{i, j}$ (see Fig. 1).

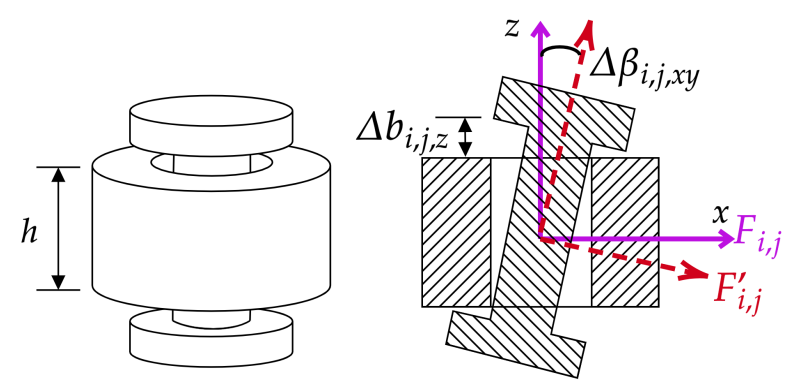

(a) $x y$ plane

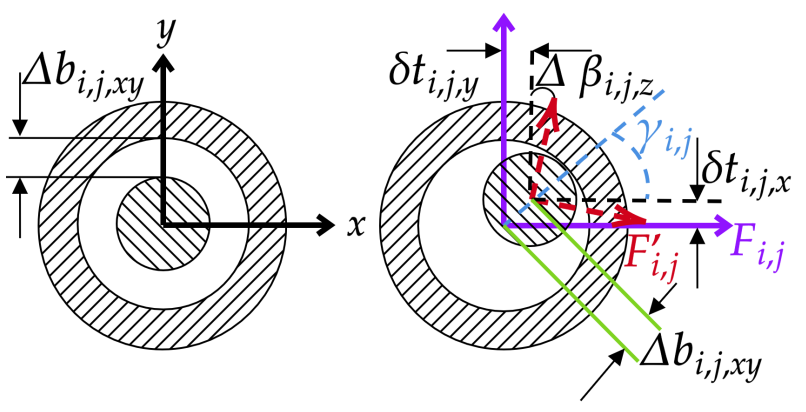

(b) $x z$ plane

Fig. 1. Joint Clearance Model.

The translational clearance along the axis joint $z$, and the rotational clearance with respect to the axis $z$ are defined as $\Delta b_{i, j, z}$ and $\Delta \beta_{i, j, x y}$, respectively. Moreover, the translational clearance in the $x y$ plane and the rotational clearance related to the $z$ axis are defined as $\Delta b_{i, j, x y}$ and $\Delta \beta_{i, j, z}$. Therefore, the elements of the error screw $\delta \mathbf{e}_{i, j}$ 
of Eq. (1) are defined by:

$$
\left\{\begin{array} { l } 
{ \delta r _ { i , j , x } = \Delta \beta _ { i , j , x y } \operatorname { c o s } ( \gamma _ { i } ) } \\
{ \delta r _ { i , j , y } = \Delta \beta _ { i , j , x y } \operatorname { s i n } ( \gamma _ { i } ) } \\
{ \delta r _ { i , j , z } = \Delta \beta _ { i , j , z } }
\end{array} \quad \left\{\begin{array}{l}
\delta t_{i, j x}=\Delta b_{i, j, x y} \cos \left(\gamma_{i}\right) \\
\delta t_{i, j x}=\Delta b_{i, j, x y} \sin \left(\gamma_{i}\right) \\
\delta t_{i, j z}=\Delta b_{i, j, z}
\end{array}\right.\right.
$$

with $0 \leq \gamma_{i} \leq 2 \pi$. Following this definition, the error pose should meet the following constraints: $\delta r_{i, j, x}^{2}+\delta r_{i, j, y}^{2} \leq \Delta \beta_{i, j, x y}^{2}$ and $\delta t_{i, j, x}^{2}+\delta t_{i, j, y}^{2} \leq \Delta b_{i, j, x y}^{2}$. The fuzzy uncertainties are introduced in the following five parameters that defines the clearances of the joints: $\Delta \beta_{i, j, z}, \Delta \beta_{i, j, x y}, \gamma_{i}, \Delta b_{i, j, x y}$ and $\Delta b_{i, j, z}$. The vector that joins the uncertain parameters of a joint clearance for a serial kinematic chain is defined as:

$$
\boldsymbol{\lambda}=\left[\begin{array}{llllll}
\boldsymbol{\lambda}_{1} & \boldsymbol{\lambda}_{2} & \ldots & \boldsymbol{\lambda}_{j} & \ldots & \boldsymbol{\lambda}_{m}
\end{array}\right]
$$

where $j=1, \ldots, n_{j}$, and $n_{j}$ is the number of joints of the kinematic chain; $\boldsymbol{\lambda}_{j}$ are the uncertain parameters of the $j-t h$ joint clearance, thus $\boldsymbol{\lambda}_{j}=$ $\left[\Delta \beta_{j, z} \Delta \beta_{j, x y} \gamma_{j} \Delta b_{j, x y} \Delta b_{j, z}\right]$.

\subsection{Error Propagation Method}

The present method for serial manipulators is based on [11]. Nevertheless, additional developments were included in the present contribution to the error propagation of the parallel manipulators.

3.2.1. Serial kinematic Chain. Initially, the Denavit-Hartenberg method is used to obtain the pose of the end effector considering no clearances. Thus, the homogeneous transformation matrix, $\mathbf{S}_{i, j}$, is defined as:

$$
\mathbf{S}_{i, j}=\left[\begin{array}{cc}
\mathbf{R}_{i, j} & \mathbf{t}_{i, j} \\
\mathbf{0}_{1 \times 3} & 1
\end{array}\right]
$$

with $i=1, \ldots, m$ and $j=1, \ldots, n_{i, f}$, respectively; $m$ is the number of kinematic chains (for a single kinematic chain $m=1$ ), and $n_{i, f}$ is the total number of frames. $\mathbf{S}_{i, j}$ represents the transformation matrix from the frame $\mathbf{F}_{i, j}$ to the frame $\mathbf{F}_{i, j+1}, \mathbf{R}_{i, j}$ is the $(3 \times 3)$ rotation matrix and $\mathbf{t}_{i, j}=\left[x_{i, j} y_{i, j} z_{i, j}\right]^{T}$ translation $(3 \mathrm{x} 1)$ vector. The pose of the end 
effector related to the $i-t h$ kinematic chain, $\mathbf{P}_{i}$, is defined as:

$$
\mathbf{P}_{i}=\prod_{j=1}^{n_{j, f}} \mathbf{S}_{i, j}
$$

However, the pose of the end effector considering the joint clearances, $\mathbf{P}_{i}^{\prime}$, will not be equal to the pose $\mathbf{P}_{i}$ presented in Eq. (4). The adjoint map transformation matrix of $\mathbf{S}_{i, j}$ maps the error screw onto the end effector at a specific pose as presented in Eq. (5).

$$
\operatorname{adj}\left(\mathbf{S}_{i, j}\right)=\left[\begin{array}{rr}
\mathbf{R}_{i, j} & \mathbf{0}_{3 \times 3} \\
\mathbf{T}_{i, j} \mathbf{R}_{i, j} & \mathbf{R}_{i, j}
\end{array}\right]
$$

where $\mathbf{T}_{i, j}=\left[\begin{array}{ccc}0 & -z_{i, j} & y_{i, j} \\ z_{i, j} & 0 & -x_{i, j} \\ -y_{i, j} & x_{i, j} & 0\end{array}\right]$ is the skew-symmetric matrix of the vector $\mathbf{t}_{i, j} \cdot \mathbf{t}_{i, j}$ and $\mathbf{R}_{i, j}$ can be extracted from the transformation matrix of Eq. (3). Moreover, The adjoint of the inverse transformation matrix, $\operatorname{adj}\left(\mathbf{S}_{i, j}\right)^{-1}$, permits to express screws at the frame $\mathbf{F}_{i, j+1}$ from $\mathbf{F}_{i, j}$.

The error screw, $\delta \mathbf{e}_{i, j}$, in the local frame $\mathbf{F}_{i, j}$, can be expressed in the end effector frame, $\mathbf{F}_{i, n_{i, f}}$, by multiplying all the inverse of the inverse adjoint transformation matrices from $n_{i, f}$ to $j+1$, thus: $\left(\prod_{k=n_{i, f}}^{j+1} \operatorname{adj}\left(\mathbf{S}_{i, k}\right)^{-1}\right) \delta \mathbf{e}_{i, j}$.

The following expression quantifies the pose error of the end effector considering all the joint clearances:

$$
\delta \mathbf{p}_{i} \mid \mathbf{F}_{i, P}=\sum_{j=1}^{n_{i}} \prod_{k=n_{i, f}}^{j+1} \operatorname{adj}\left(\mathbf{S}_{i, k}\right)^{-1} \delta \mathbf{e}_{i, j}
$$

with $n_{i}$ being the number of joints, and $n_{i, f}$ the number of frames; note that $n_{i, f} \geq n_{i}$. $\delta \mathbf{p}_{i} \mid \mathbf{F}_{i, P}$ is the pose error in the frame attached to the end effector $\mathbf{F}_{i, P}$.

The pose error in the end effector $\delta \mathbf{p}_{i} \mid \mathbf{F}_{i, P}$ at the frame $\mathbf{F}_{i, P}$ should be expressed in the reference frame attached to the fixed base $\mathbf{F}_{i, 1}$ by introducing the matrix $\mathbf{N}_{i, j}$. Thus,

$$
\delta \mathbf{p}_{i}\left|\mathbf{F}_{i, 1}=\prod_{j=1}^{n_{i, f}}\left(\mathbf{N}_{i, j}\right) \delta \mathbf{p}_{i}\right| \mathbf{F}_{i, P}
$$


where $\mathbf{N}_{i, j}=\left[\begin{array}{cc}\mathbf{R}_{i, j} & \mathbf{0}_{3 \times 3} \\ \mathbf{0}_{3 \times 3} & \mathbf{R}_{i, j}\end{array}\right]$ maps the orientation error and the translational error from the frame $\mathbf{F}_{i, P}$ to the frame $\mathbf{F}_{i, 1}$. Therefore, an expression for $\delta \mathbf{p}_{i} \mid \mathbf{F}_{i, 1}$ is obtained by substituting Eq. (6) into Eq. (7).

$$
\delta \mathbf{p}_{i} \mid \mathbf{F}_{i, 1}=\sum_{j=1}^{n_{i}} \prod_{l=1}^{n_{i, f}}\left(\mathbf{N}_{i, l}\right) \prod_{k=n_{i, f}}^{j+1} \operatorname{adj}\left(\mathbf{S}_{i, k}\right)^{-1} \delta \mathbf{e}_{i, j}
$$

The expression of Eq. (8) can be written in the following compact form:

$$
\delta \mathbf{p}=\mathbf{M}_{i} \delta \mathbf{e}_{i}=\left[\delta \mathbf{p}_{r} \delta \mathbf{p}_{t}\right]^{T}
$$

where $\mathbf{M}_{i}=\left[\mathbf{M}_{i, 1} \ldots \mathbf{M}_{i, n i}\right], \delta \mathbf{e}_{i}=\left[\delta \mathbf{e}_{i, 1}^{T} \ldots \delta \mathbf{e}_{i, n_{i}}^{T}\right]$

$\mathbf{M}_{i, j}=\prod_{l=1}^{n_{i, f}}\left(\mathbf{N}_{i, l}\right) \prod_{k=n_{i, f}}^{j+1}\left(\operatorname{adj}\left(\mathbf{S}_{i, k}\right)^{-1}\right), \delta \mathbf{p}_{r}$ and $\delta \mathbf{p}_{t}$ are the orientation and translational error of the end effector, respectively.

3.2.2. Parallel Mechanism. Parallel manipulators are composed of several kinematic chains that connect a fixed base to a movable platform; nevertheless, the approach proposed in the present contribution is limited to parallel manipulator with identical kinematic chains. Differently of serial manipulators, the parallel manipulators are subject to kinematic constraints introduced by their closed-loop kinematic configurations. These kinematic constraints must be considered to propagate the errors of the joint clearance onto the end effector. Moreover, the pose obtained from any kinematic chain should be equal to each other, therefore $\mathbf{P}_{1}=\mathbf{P}_{2} \cdots=\mathbf{P}_{m}$.

The errors of the joints are correlated due to the kinematic constraints of the parallel mechanism. The following assumptions are considered:

1. The errors of all the joint clearances of each kinematic chain defined by $\delta \mathbf{e}_{i}$ are correlated. This correlation depends on the orientation of the local frames at the base point of the kinematic chains, $\mathbf{R}_{i}$, concerning the fixed frame. Thus, $\delta \mathbf{e}_{i}=$ $\mathbf{Q}_{i}\left[\delta \mathbf{e}_{i, 1}^{T} \ldots \delta \mathbf{e}_{i, n_{i}}^{T}\right]$ with $\mathbf{Q}_{i, j}=\left[\begin{array}{cc}\mathbf{R}_{i} & \mathbf{0}_{3 \times 3} \\ \mathbf{0}_{3 \times 3} & \mathbf{R}_{i}\end{array}\right]$.

2. The passive joints are free to move along the axisymmetric axis. Therefore no clearance around the axial axis is considered for the passive joints, thus $\Delta \beta_{i, j, z}=0$. 
3. The errors of the prismatic or revolute active joints are entirely independent. They are defined by $\Delta \mathbf{q}=\left[\Delta q_{1} \ldots \Delta q_{m}\right]^{T}$.

The errors of the joint clearances are propagated for every single kinematic chain by using the expression of Eq. (8), and the error produced by the active joints is also considered by using the Jacobian matrix $\mathbf{J}$.

$$
\delta \mathbf{p}_{i}=\mathbf{W} \mathbf{J}^{-1} \delta \Delta \mathbf{q}+\mathbf{M}_{i} \delta \mathbf{e}_{i}
$$

where $\mathbf{W}$ transforms the end effector error to an error screw. The definition of this matrix will depend on the kinematics of the parallel mechanism. The minor error along each Cartesian coordinate of the errors of every kinematic chain $\delta \mathbf{p}_{i}$ is considered in order to respect the kinematic constraints of the parallel mechanism $\left(\mathbf{P}_{1}=\mathbf{P}_{2} \cdots=\mathbf{P}_{m}\right)$. Thus, the total error in the end effector of the parallel mechanism $\delta \mathbf{p}$ is defined by the following expression.

$$
\delta \mathbf{p}=\min \left(\delta \mathbf{p}_{1} \delta \mathbf{p}_{2} \ldots \delta \mathbf{p}_{m}\right)=\left[\delta \mathbf{p}_{r} \delta \mathbf{p}_{t}\right]^{T}
$$

where $\delta \mathbf{p}_{r}$ and $\delta \mathbf{p}_{t}$ are the orientation and translational error of the end effector, respectively.

The clearances of the spherical joints can also be included in the proposed approach based on the axisymmetric model of the joint clearance presented in Fig. 1. For this purpose, the spherical joint should be represented by three intersecting revolute joints as presented in [22]. Then, the clearances are modeled in the revolute joints by using the axisymmetric model of sec. 3.1 .

\subsection{Fuzzy Kinematic Reliability Method}

This method aims at computing the fuzzy reliability index to assess the kinematic accuracy of a mechanism subject to an uncertain error produced by the joint clearances. The proposed approach is based on the fuzzy uncertainty theory.

3.3.1. Definitions of Fuzzy Concepts. Let $\mathbf{K}$ be an universal set of objects whose generic elements are denoted by $k$. A fuzzy parameter $\tilde{\mathbf{k}}$ is defined by the membership function $\mu_{k}: \mathbf{K} \rightarrow[0,1]$, where $[0,1]$ is a continuous interval. The membership function, $\mu_{k}$, establishes how compatible is the element $k$ to $\tilde{\mathbf{k}}$; i.e. the more belonging of $k$ to $\tilde{\mathbf{k}}$ 
implies that the membership function $\mu_{k}(k)$ get values close to "1" (see Fig 2(a)). The determinstic definition of the parameter $k_{d}$ (where, $k \in \mathbf{K}$ ) is defined by the classical membership function $\mu_{k}: \mathbf{K} \rightarrow\{0,1\}$, where $\mu_{k}=1$ specifically for $k_{d}$, otherwise $\mu_{k}=0$ (see Fig 2(a)).

$$
\tilde{\mathbf{k}}=\left\{\left(k, \mu_{k}(k)\right) \mid k \in \mathbf{K}\right\}
$$

where $0 \leq \mu_{k} \leq 1$

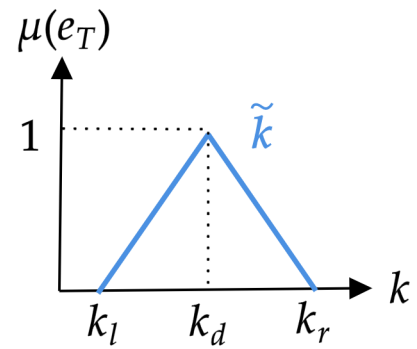

(a) fuzzy parameter.

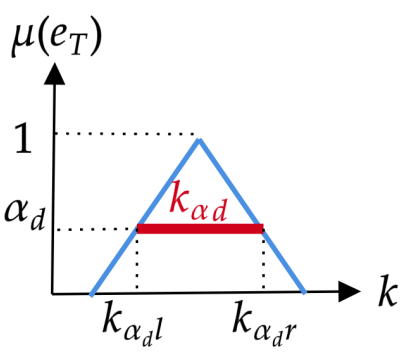

(b) $\alpha$-level representantion

Fig. 2. Fuzzy definitions.

The fuzzy parameter $\tilde{\mathbf{k}}$ can be represented by the intervals $k_{\alpha_{d}}$ weighted by the membership function $\mu_{k}$; these intervals are denominated as $\alpha$-levels and this representation is suitable for computational purposes (see Fig. 2(b)); thus:

$$
k_{\alpha_{d}}=\left\{k \in \mathbf{K}, \mu_{k}(k) \geq \alpha_{d}\right\}
$$

The $\alpha$-level interval $k_{\alpha_{d}}=\left[k_{\alpha_{d} l}, k_{\alpha_{d}}\right]$ for the unidimensional fuzzy variables presented in the Fig. 2 where:

$$
\begin{aligned}
& k_{\alpha_{d} l}=\min \left[\left\{k \in \mathbf{K}, \mu_{k}(k) \geq \alpha_{d}\right\}\right] \\
& k_{\alpha_{d} r}=\max \left[\left\{k \in \mathbf{K}, \mu_{k}(k) \geq \alpha_{d}\right\}\right]
\end{aligned}
$$

The error of the end effector is evaluated by using the method presented in section 3.2 ; the error, $e_{T}$, is obtained based on the Eqs. (9) and (11) for serial and parallel manipulators, respectively. It is worth mentioning that the error, $e_{T}$, can consider the orientation error $\left(e_{T}=\left\|\delta \mathbf{p}_{r}\right\|\right)$ or the translational error $\left(e_{T}(\mathbf{c})=\left\|\delta \mathbf{p}_{t}\right\|\right)$ separately where $\|$.$\| represents the magnitude of the vector. The uncertain parameters of the joint$ 
clearances are defined as fuzzy variables; thus: $\tilde{\boldsymbol{\lambda}}_{j}=\left[\Delta \tilde{\beta}_{j, z} \Delta \tilde{\beta}_{j, x y} \tilde{\gamma}_{j} \Delta \tilde{b}_{j, x y} \Delta \tilde{b}_{j, z}\right]$ based on the definition of Eq. (12). Therefore, the error will also be a fuzzy uncertain variable. The uncertain fuzzy error $\tilde{\mathbf{e}}_{T}$ is defined by using the $\alpha$-level representation:

$$
\tilde{\mathbf{e}}_{T}=\left\{\left(e_{T}, \mu(e)\right) \mid e_{T} \in \mathbb{R}_{>0}\right\} \quad \text { where } 0 \leq \mu\left(e_{T}\right) \leq 1
$$

Moreover, the fuzzy error $\tilde{\mathbf{e}}_{T}$ can be represented by $\alpha$-levels, thus $e_{\alpha_{k}}=\left\{e_{T} \in\right.$ $\left.\mathbb{R}_{>0}, \mu\left(e_{T}\right) \geq \alpha_{k}\right\}$. Alternatively, $e_{\alpha_{k}}$ can be defined as an interval weighted by the membership function $\mu\left(e_{T}\right)$, thus:

$$
e_{\alpha_{k}}=\left(0, e_{\alpha_{k} r}\right)
$$

where $e_{\alpha_{k} r}=\max \left(e_{T} \in \mathbb{R}_{>0}, \mu\left(e_{T}\right) \geq \alpha_{k}\right)$. The evaluation of $e_{\alpha_{k} r}$ demands the solution of an optimization problem to determine the upper limit of the uncertain error corresponding to the $\alpha_{k}$ value of the $\mu\left(e_{T}\right)$ membership function.

3.3.2. Fuzzy Reliability Index. The possibility of the fuzzy error, $\tilde{\mathbf{e}}_{T}$, exeeds the maximum error limit $e_{\max }$ is quantified by the fuzzy kinematic reliability, $\alpha_{f}$, thus:

$$
\alpha_{f}=\mu\left\{\tilde{\mathbf{e}}_{T}>e_{\max }\right\}
$$

where $\mu\{$.$\} represents the possibility that is quantified by the membership function value$ $\alpha_{f}$ with $0 \leq \alpha_{f} \leq 1$.

The fuzzy kinematic reliability aims at determining the possibility of failure by examining the fuzzy error. Figure 3.3.2 shows that a failure is produced when $\tilde{e}>e_{\max }$. For this condition, the possibility of failure is given by $\alpha_{f}$.

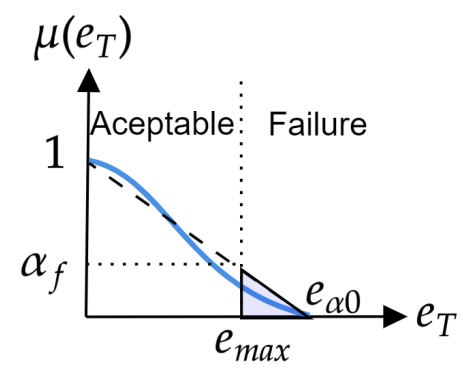

Fig. 3. Fuzzy error, $\tilde{\mathbf{e}}_{T}$, and linear approximation to estimate $\alpha_{f}$. 
For this particular application, the membership function of the fuzzy error $\mu\left(\tilde{\mathbf{e}}_{T}\right)$ is approximated as a linear function in order to estimate the failure possibility considering the maximum position error $e_{\alpha 0}$ only. This linear approximation is the simplest form to compute the possibility $\mu\{$.$\} of Eq. (16) to obtain the failure possibility \alpha_{f}$; however, high order functions could be used to enhance the approximation of $\alpha_{f}$. If the fuzzy error exeeds tha maximum error limint, i.e. if the fuzzy error surpass $e_{\max }$ and it attains the failure region (see Fig. 3.3.2), the following expression computes the linear estimation of the failure possibility:

$$
\alpha_{f}=\frac{e_{\alpha_{0}}-e_{\max }}{e_{\alpha_{0}}}
$$

otherwise, if fuzzy error is less than $e_{\max }$, the fuzzy error is confined in the aceptable region (see Fig. 3.3.2), then $\alpha_{f}=0$. Moreover, $e_{\alpha_{0}}$ which is the uncertain fuzzy error $\tilde{\mathbf{e}}_{T}$ evalueated at the $\alpha$-level $\alpha_{k}=0$ is obtained by solving the following maximization:

$$
e_{\alpha_{0}}=\max _{\boldsymbol{\lambda}_{\alpha_{0}}} e(\boldsymbol{\lambda})
$$

where $\boldsymbol{\lambda}=\left[\begin{array}{llll}\boldsymbol{\lambda}_{1} \ldots & \boldsymbol{\lambda}_{j} \ldots & \boldsymbol{\lambda}_{n_{i, f}}\end{array}\right]$ defined in Eq. (2) and $\boldsymbol{\lambda}_{\alpha_{0}}=\left[\boldsymbol{\lambda}_{l}, \boldsymbol{\lambda}_{r}\right]$ is the crisp space at $\alpha_{k}=0$. The optimization problem of Eq. (18) could be solve to compute the failure possibility $\alpha_{f}$ of Eq. (17); this optimization was solved by using the Differential Evolution (DE) algorithm [21].

The failure possibility, $\alpha_{f}$, is bounded as follows:

$$
0 \leq \alpha_{f} \leq 1
$$

where the desired performance of a manipulator consists of minimizing the failure possibility. It is worth mentioning that the failure possibility indicates the percentual rate of the maximum fuzzy error $\tilde{\mathbf{e}}_{T}$ surpassing the maximum admissible error $e_{\max }$, e.g. $\alpha_{f}=0.45$ indicates that the maximum fuzzy error overpass $45 \%$ the maximum admissible error $e_{\max }$.

Finally, the algorithm to compute the fuzzy kinematic reliability is illustrated in the flow chart of Fig. 4. The kinematic reliability is computed based on the differential evolution (DE) algorithm to find the maximum error $e_{\alpha 0}$ as presented in Fig. 4(a). Initially, a set of candidate of solutions $\alpha_{p l}$ is generated by using the DE operators. The 
positioning error $e_{T}$ is evaluated for these candidate solutions and the candidate solutions are optimized for several iterations until meeting the stopping criteria. As presented in Fig. 4(b), the positioning error $e_{T}$ produced by the joint clearances is obtained based on the uncertain parameters $\boldsymbol{\lambda}$ of the joint defined in Eq. (2).

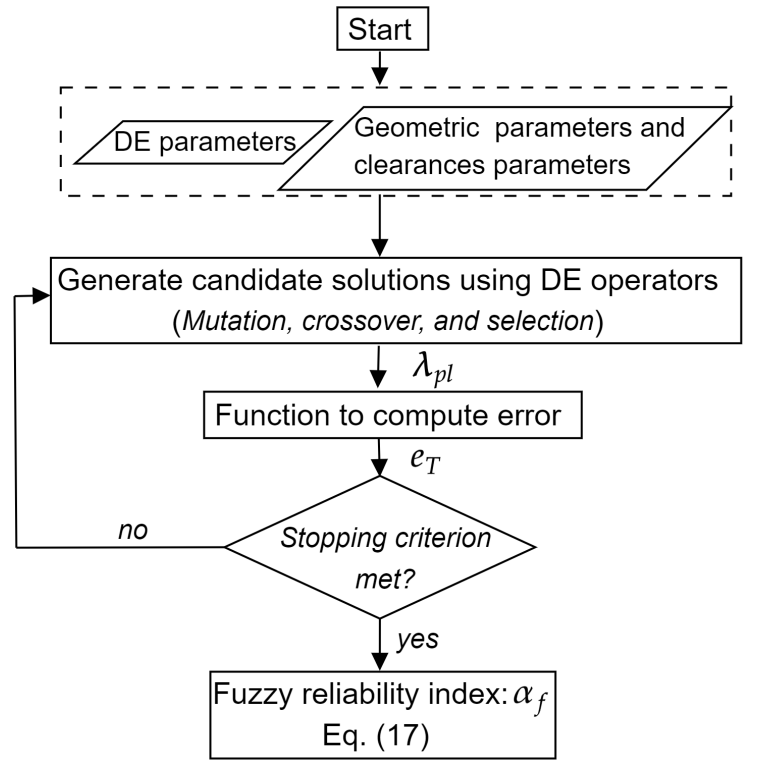

(a) kinematic reliability method.

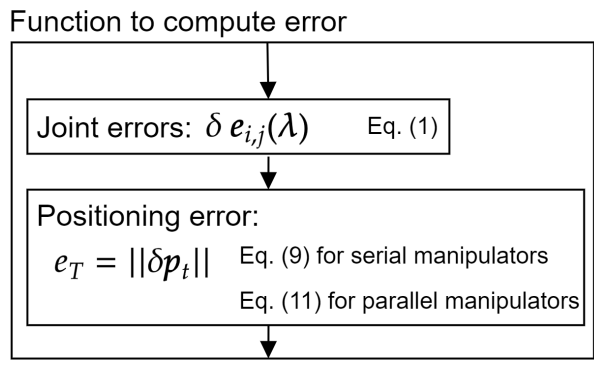

(b) Function to compute error.

Fig. 4. Flow chart of the fuzzy kinematic reliability method.

\section{Numerical Applications}

The fuzzy kinematic reliability of a serial 3R manipulator, and a Cartesian parallel manipulator $(\mathrm{CPM})$ is analyzed in this section.

Initially, the fuzzy parameters of axisymmetric joint clearances of the manipulators are defined. The parameters of the active joint clearances, according to the description of Fig. 2, are defined as triangular fuzzy variables, thus $\tilde{\beta}_{x y}=<0,0.05,0.1>^{\circ}$, $\tilde{\beta}_{z}=<0,0.025,0.05>^{\circ}, \tilde{b}_{x y}=<0,0.5,1>\times 10^{-4} \mathrm{~m}, \tilde{b}_{z}=<0,0.5,1>\times 10^{-4} \mathrm{~m}$, and $\tilde{\gamma}=<$ $0,180,360>^{\circ}$. The passive joints have same parameters; nevertheless, no clearance around the axial axis is considered, thus $\tilde{\beta}_{z}=0$. Therefore, the translational error us consider in this analysis, thus $e_{T}=\left\|\delta \mathbf{p}_{t}\right\|$, and $e_{\max }=1 \times 10-3 \mathrm{~m}$ to evaluate the kinematic reliability.

The parameters selected to run the DE algorithm were: population size is -5 per uncertain variable, 40 generations, crossover probability rate is -0.8 , perturbation rate is -0.8 and the $D E /$ rand $/ 1 /$ bin strategy for the mutation mechanism. 
The Monte Carlo simulation (MCS) is also used to compute the kinematic reliability using a probabilistic approach. The algorithm to estimate the kinematic reliability by using the Monte Carlo simulation is presented in the appendix A.

Finally, the following hardware was used: i7 Intel i7-7500U CPU processor $(2.9 \mathrm{GHz})$ and RAM 8.0 GB.

\subsection{R Serial Manipulator}

The 3R serial arm is presented in Fig. 5, and its D-H parameters are defined in Tab. I. Moreover, the maximum admissible error is defined as $e_{\max }=1 \times 10^{-3} \mathrm{~m}$ and the number of samples to compute the Monte Carlo simulation is $n_{s}=1.5 \times 10^{5}$ according to the convergence analyis presented in the appendix B.

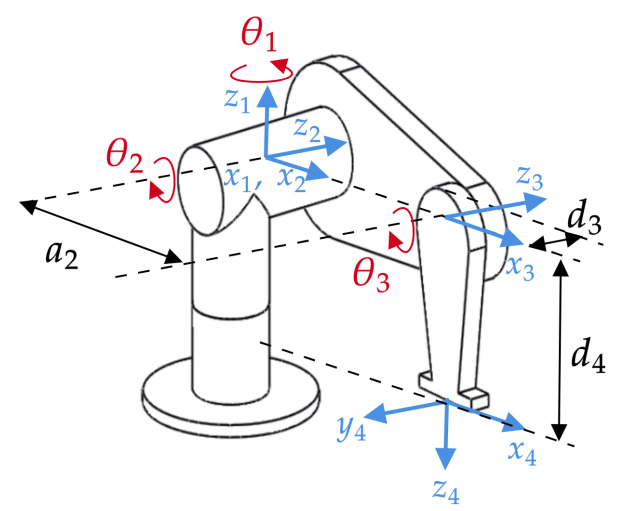

Fig. 5. 3R serial manipulator.

According to the error propagation method of section 3.2.1, $i=1$ and $j=1, \ldots, 3$. The link lengths and maximum limits of the rotational joints are defined as $a_{2}=0,15 \mathrm{~m}$, $d_{3}=0,01 \mathrm{~m}, d_{4}=0,10 \mathrm{~m},-100^{\circ} \leq \theta_{1} \leq 90^{\circ},-90^{\circ} \leq \theta_{2} \leq 45^{\circ}$ and,$-90^{\circ} \leq \theta_{3} \leq 90^{\circ}$.

Table I. D-H parameters of 3R manipulator..

\begin{tabular}{lllll}
\hline$j$ & $\alpha_{j-1}$ & $a_{j-1}$ & $d_{j}$ & $\theta_{j}$ \\
\hline 1 & 0 & 0 & 0 & $\theta_{1}$ \\
2 & $-90^{\circ}$ & 0 & 0 & $\theta_{2}$ \\
3 & 0 & $a_{2}$ & $d_{3}$ & $\theta_{3}$ \\
4 & $-90^{\circ}$ & 0 & $d_{4}$ & 0 \\
\hline
\end{tabular}

Initially, the propagation error method proposed in section 3.2.1 is evaluated. The parameters of the active joint clearances are defined as $\beta_{x y}=0.05^{\circ}, \beta_{z}=0.025^{\circ}, b_{x y}=$ $0.5 \times 10^{-4} \mathrm{~m}, b_{z}=0.5 \times 10^{-4} \mathrm{~m}$, and $\gamma=180^{\circ}$. The orientation and translational error for the $x y$ plane of the workspace is presented in Fig. 6. It is observed that the error 
is amplified in the lowest region of the workspace for this specific definition of the joint clearances.

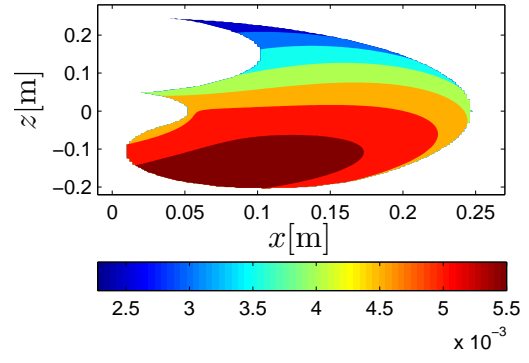

(a) Orientation error.

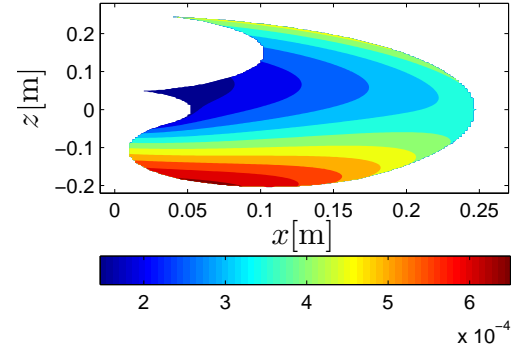

(b) Translational error.

Fig. 6. Error over the $x z$ plane of the workspace.

The error is also computed at the Cartesian positioning of the end effector $\mathbf{p}=$ $\left[\begin{array}{lll}0.2 & 0 & 0\end{array}\right]^{T}$ by using the fuzzy approach and the Monte Carlo simulation (see Fig. 7). According to the definition of the fuzzy parameters of the clearances $\tilde{\lambda}$, the maximum possibility $\mu\left(e_{T}\right)=1$ corresponds to a null error $\left.\mu\left(e_{T}\right)\right)=0 \mathrm{~m}$, i.e. it is expected a null positioning error and the positioning error increases with the decrease of the possibility $\mu\left(e_{T}\right)$ (see Fig. $\left.7(\mathrm{a})\right)$. Moreover, it is observed that the linear approximation fits the membership function $\mu\left(e_{T}\right)$ as presented the Eq. (15), this approximation is useful to compute the fuzzy kinematic reliability. The probability density function (PDF) was also estimated based on the results obtained by using the MSC (see Fig. 7(b)).

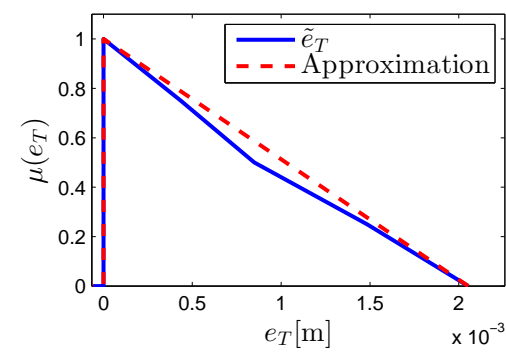

(a) Error derived from the fuzzy approach.

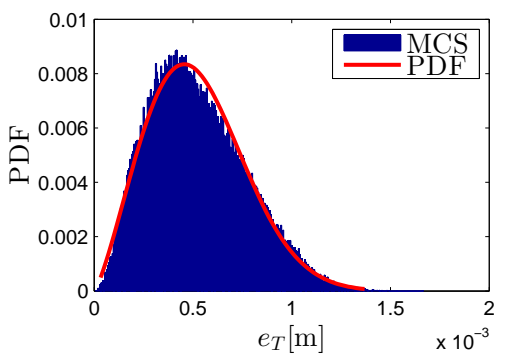

(b) Error derived from the MCS.

Fig. 7. Error evaluation using the fuzzy approach and MCS.

Then, the kinematic dexterity based on the condition number of the Jacobian matrix is also computed to evaluate the performance of the manipulator over the usable workspace as presented in Fig. 8. The local kinematic dexterity corresponds to $1 / \kappa(\mathbf{J})$, where $\kappa($. is the condition number of a matrix, and $\mathbf{J}$ is the Jacobian matrix.

The kinematic reliability is also evaluated over the usable workspace using the Monte Carlo simulation as shown in Fig. 9. The failure probability, $p_{f}$, increases in the outer 


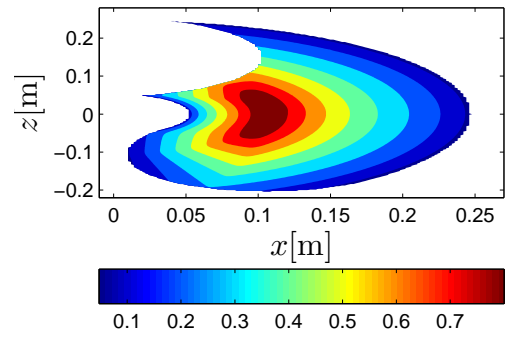

(a) $x z$ plane.

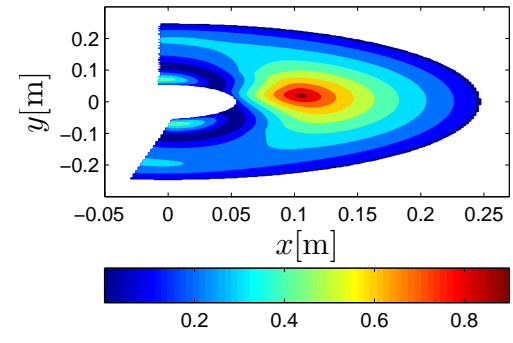

(b) $x y$ plane.

Fig. 8. $1 / k(\mathbf{J})$ over the usable workspace.

limit of the usable workspace that corresponds to poses in which the $3 R$ manipulator is extended. The increment of $p_{f}$ is produced by the increasing of the kinematic error in the outer borders of the usable workspace. The left side of the usable workspace corresponds to poses in which the manipulator is retract; therefore, the kinematic error and $p_{f}$ decreases (see Figs. 9(a) and 9(b)). For this specific manipulator, it is observed an inverse relationship between kinematic reliability (see Fig. 9) and kinematic dexterity (see Fig. 8) for the poses in which the manipulator is extended, i.e. $p_{f}$ increases and $1 / \kappa(\mathbf{J})$ decreases. Nevertheless, this behavior is not observed for the poses in which the manipulator is retracted.

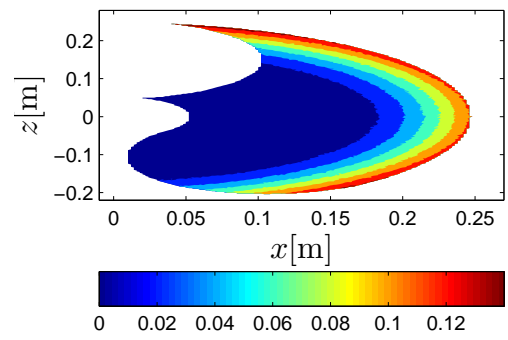

(a) $x z$ plane.

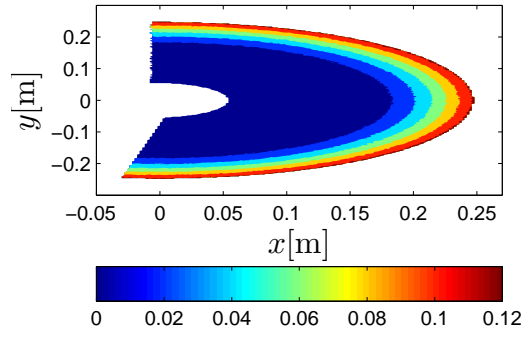

(b) $x y$ plane.

Fig. 9. $p_{f}$ over the usable workspace.

Finally, the fuzzy kinematic reliability is computed and analyzed (see Fig. 10). The possibility of failure, $\alpha_{f}$, over the $x y$ plane of the workspace is presented in Fig. 10. $\alpha_{f}$ increases for the regions of workspace in which the manipulator is extended and therefore the maximum fuzzy error also increases; these poses located in the border of the right side. Additionally, it is observed that the possibility of failure has similar behavior with the probability of failure estimated with the MCS method (see Figs. 9 and 10). 


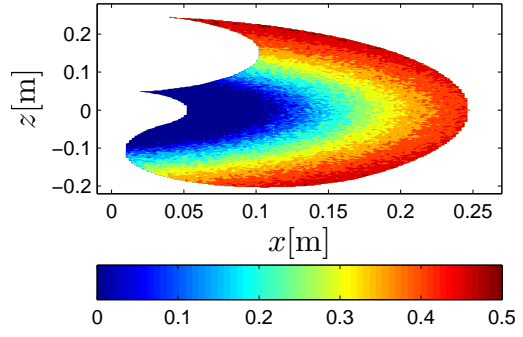

(a) $x z$ plane.

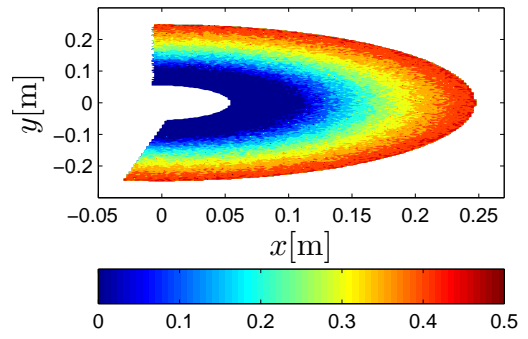

(b) $x y$ plane.

Fig. 10. $\alpha_{f}$ over the usable workspace.

\subsection{Cartesian Parallel Manipulator}

The Cartesian Parallel Manipulator (CPM) has three symmetric kinematic chains that joint the moving platform $P$ to the fixed frame (see Fig. 11). The three active prismatic joints $\left(\mathbf{q}=\left[\begin{array}{lll}q_{1} & q_{2} & q_{3}\end{array}\right]^{T}\right)$ act along the $X, Y$, and $Z$ axes. The moving platform has three translational degrees of freedom defined by $(x, y, z)$. Every kinematic chain is located at the frame $O_{j}$, and it has three passive rotational joints defined by $\theta_{j, i}$, for $i=1,2,3$, and $j=1,2,3$. The link's lengths of every kinematic chain are defined by $l_{1}, l_{2}$, and the geometry of the moving platform is defined by $l_{p}$. The active joints specify the Cartesian position of the moving platform directly, thus $q_{1}=x, q_{2}=y$, and $q_{3}=z$. Therefore, the Jacobian matrix is a $3 \times 3$ identity matrix, $\mathbf{J}=\mathbb{I}_{3 \times 3}$.

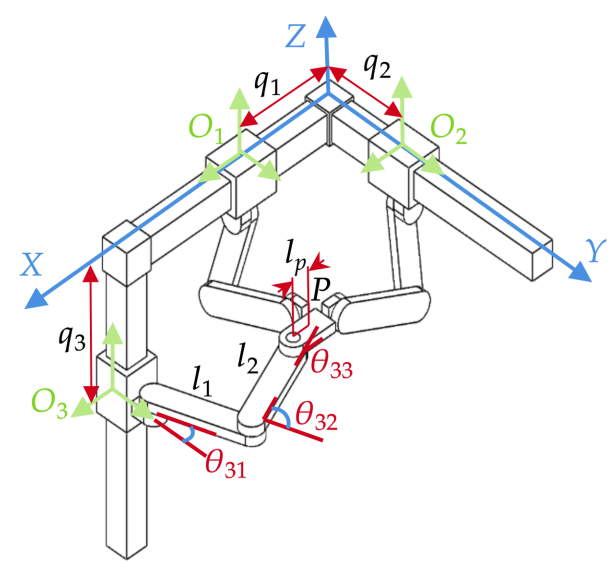

Fig. 11. Cartesian Parallel Manipulator (CPM).

The D-H parameters of the $j-t h$ kinematic chain from the frame $O_{j}$ to $P$ are presented in table II.

For this numerical application the geometric parameters of the manipulator and the clearances were defined as follows. The link's length was defined specifically as $l_{1}=$ $0.077 \mathrm{~m}, l_{2}=0.077 \mathrm{~m}$ and $l_{p}=0.022 \mathrm{~m}$. A fuzzy triangular position error was considered 
Table II . D-H parameters of Cartecian parallel manipulator..

\begin{tabular}{lllll}
\hline$j$ & $\alpha_{j-1}$ & $a_{j-1}$ & $d_{j}$ & $\theta_{j}$ \\
\hline 1 & 0 & 0 & 0 & $\theta_{j, 1}$ \\
2 & 0 & $l_{1}$ & 0 & $\theta_{j, 2}$ \\
3 & 0 & $l_{2}$ & 0 & $\theta_{j, 3}$ \\
$P$ & 0 & $l_{p}$ & 0 & 0 \\
\hline
\end{tabular}

for every prismatic active joint $\Delta \tilde{q}_{j}=<0,0.5,1>\times 10^{-4} \mathrm{~m}$. Moreover, the maximum admissible error is defined as $e_{\max }=2.5 \times 10^{-4} \mathrm{~m}$.

The orientation of the kinematic chains $\mathbf{Q}_{i}$ is defined by the orientation matrices: $\mathbf{R}_{1}=\mathbb{I}_{3 x 3}, \mathbf{R}_{2}=\mathbf{R}\left(90^{\circ}, X\right)$ and $\mathbf{R}_{3}=\mathbf{R}\left(90^{\circ}, Y\right)$. According to Eq. (10), the position error is evaluated with $\mathbf{W}=\left[\mathbf{0} \mathbb{I}_{3 x 3}\right]^{T}$. The position error of $P, \delta \mathbf{p}(\theta)$, at the end effector position $[0.0690 .0890 .089] \mathrm{m}$.

Initially, the propagation error method for parallel manipulators of section 3.2.2 is analyzed. The parameters of each of the prismatic active joint is $\Delta q_{j}=0.5 \times 10^{-4} \mathrm{~m}$. The parameters of each passive joint clearances is defined as $\beta_{x y}=0.05^{\circ}, \beta_{z}=0.025^{\circ}$, $b_{x y}=0.5 \times 10^{-4} \mathrm{~m}, b_{z}=0.5 \times 10^{-4} \mathrm{~m}$, and $\gamma=180^{\circ}$. The translational error $e_{T}$ for the $x y$ plane and the $x y$ plane of the workspace is presented in Fig. 12.

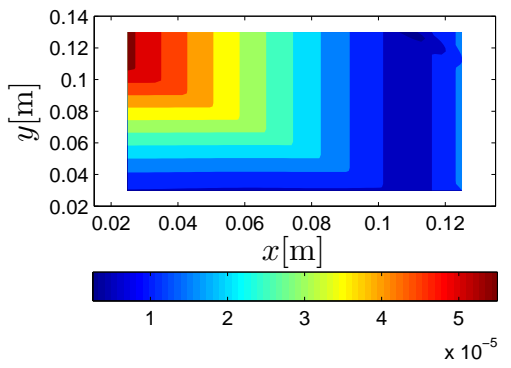

(a) $x y$ plane.

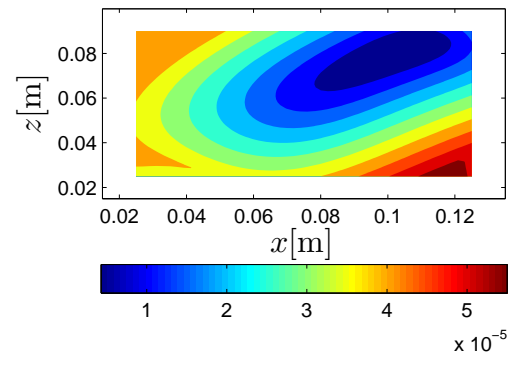

(b) $x z$ plane.

Fig. 12. Translational $e_{T}$ error over the workspace of the CPM.

Figure 13(a) presents the fuzzy translational error $\tilde{e}_{T}$. The failure possibility $\alpha_{f}$ estimated with the linear approximation of Eq. (17) is 0.5142 and evaluated $\tilde{g}$ is 0.4940 (see Fig. 13(a)); the percentage difference between these results is about 4.08\%; therefore, the linear approximation of $\alpha_{f}$ is valid. The obtained $\alpha_{f}$ means $51.42 \%$ of the possibility that position error exceeds the maximum admissible limit. Moreover, the kinematic reliability was also evaluated using the Monte Carlo simulation as presented in Fig. 13(b). Thus, the failure probability was estimated as $p_{f}=0.045$; This probability means the ratio between all the computed errors and the error outside the maximum limit sphere (see Fig. 13(b)). 


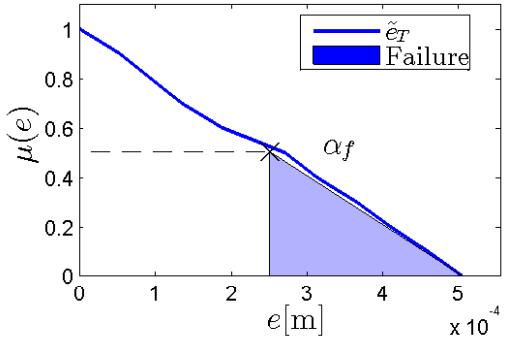

(a) $\tilde{\mathbf{g}}$.

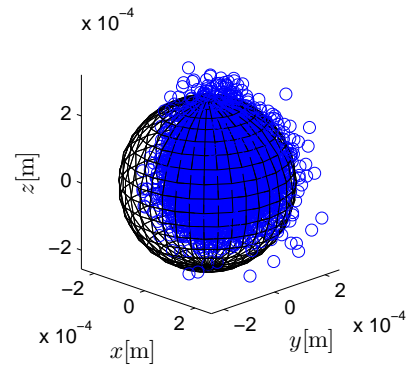

(b) Reliability analysis using MCS method.

Fig. 13. Position error of $P$.

The kinematic reliability of the CPM was estimated using the Monte Carlo method over two planes of the workspace as shown in Fig.14. The failure probability, $p_{f}$, increases in the regions of the workspace where in the uncertain kinematic error augments.

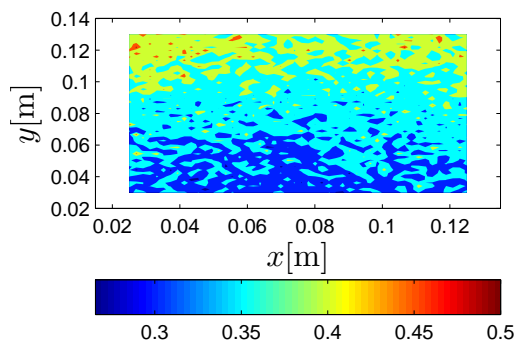

(a) $x y$ plane.

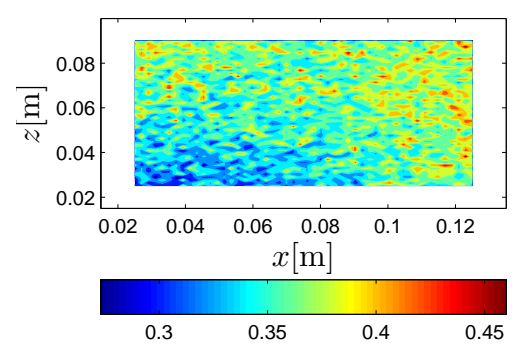

(b) $x z$ plane.

Fig. 14. Kinematic reliability over the workspace of the CPM.

The possibility of failure $\alpha_{f}$ is presented in Fig. 15. $\alpha_{f}$ increases for the regions of workspace in which all the kinematic chains are extended, i.e., the extension of kinematic chain increases position error and $\alpha_{f}$. Moreover, one can observe that the possibility of failure has similar behavior with the probability of failure estimated with the MCS method (see Fig. 14), i.e., the possibility and probability of failure increase and decrease in the same regions of the workspace.

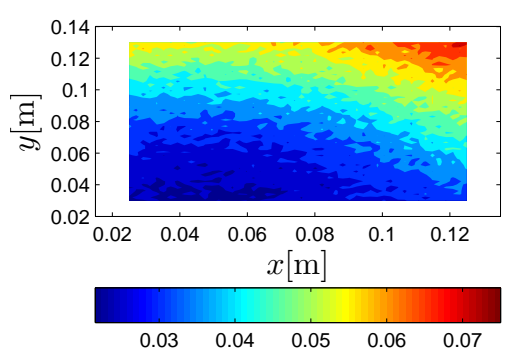

(a) $x y$ plane.

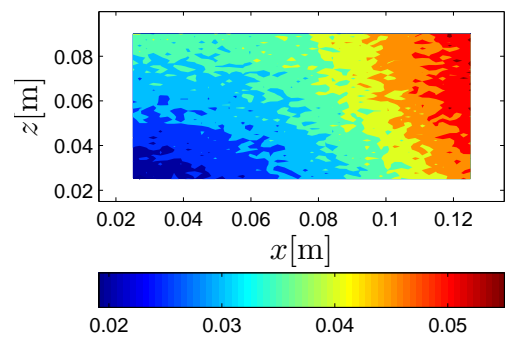

(b) $x z$ plane.

Fig. 15. $\alpha_{f}$ over the workspace of the CPM: 


\subsection{Computational Complexity}

The algorithm of the fuzzy reliability is based on the computation of the positioning error $\left\|\delta \mathbf{p}_{t}\right\|$ as presented in the algorithm of Fig. 4. Moreover, the reliability algorithm to compute the probability of failure described in the appendix $\mathrm{A}$ is also based on the computation of positioning error. Therefore, the computational complexity of the fuzzy kinematic reliability algorithm is analyzed in terms of number of positioning error evaluations $(n e)$ and the computational time $\left(t_{c}\right)$.

A comparison is presented between the fuzzy approach and the Monte Carlo Method in Tab. III for the Cartesian parallel manipulator. Thus, three Cartesian positions of the end effector are considered for this analysis.

Table III . Computational complexity of the fuzzy reliability algorithm.

\begin{tabular}{c|cc|cc}
\hline & \multicolumn{2}{|c|}{ Fuzzy Method } & \multicolumn{2}{c}{ MCS } \\
$\mathbf{p}[\mathrm{m}]$ & ne & $t_{c}[\mathrm{~s}]$ & $n e$ & $t_{c}[\mathrm{~s}]$ \\
\hline $\mathbf{p}_{1}=\left[\begin{array}{lll}0.04 & 0.04 & 0.04\end{array}\right]^{T}$ & 3198 & 5.3855 & $1.5 \times 10^{5}$ & 4.1137 \\
$\mathbf{p}_{2}=\left[\begin{array}{lll}0.08 & 0.08 & 0.06\end{array}\right]^{T}$ & 3198 & 5.3844 & $1.5 \times 10^{5}$ & 4.0995 \\
$\mathbf{p}_{3}=\left[\begin{array}{lll}0.12 & 0.12 & 0.08\end{array}\right]^{T}$ & 3198 & 5.3853 & $1.5 \times 10^{5}$ & 4.1770 \\
\hline
\end{tabular}

The results show that the fuzzy method demands the computation of positioning error fewer times than the Monte Carlo Simulation. Nevertheless, the operations of the differential evolution (DE) optimization (mutation, crossover, and selection) increase the computational time when comparing to the Monte Carlo simulation.

\section{Conclusion}

The proposed fuzzy reliability method permitted to compute the kinematic failure possibility of manipulators as an alternative to the probabilistic approaches widely used in the literature. Moreover, the proposed approach allows quantifying a kinematic reliability index that takes into account the kinematic constraints and the effect of clearances on the kinematic chains of serial and parallel manipulators.

The kinematic criteria based on the condition number of the Jacobian matrix will not reveal enough information about the effects of clearances on the kinematic accuracy of the manipulator. The kinematic reliability demonstrated an alternative criterion that takes directly into account the effects of errors and uncertainty simultaneously. Moreover, the fuzzy kinematic reliability computes a ratio of uncertain error surpassing a maximum admissible limit; this information could be used for the optimal design of manipulators. 
Future work will encompass the optimal design of the mechanism based on the proposed fuzzy kinematic reliability method.

\section{Acknowledgements}

The authors are thankful for the financial support provided by $\mathrm{CNPq}$ (Process 427204/2018-6), and CAPES.

\section{References}

1. F.A. Lara-Molina, D. Dumur and K.A. Takano, "Multi-objective optimal design of flexible-joint parallel robot" Engineering Computations 35(8), pp. 2775-2801 (2018).

2. B. Siciliano and O. Khatib, Springer Handbook of Robotics (Springer-Verlag. Berlin, Heidelberg, 2007).

3. J. Takahashi, T. Fukukawa and T. Fukuda, "Passive Alignment Principle for Robotic Assembly between a Ring and a Shaft with Extremely Narrow Clearance," IEEE/ASME Trans. on Mechatronics 21(1), 196-204 (2016). chebbi2009prediction

4. A.-H. Chebbi, Z. Affi and L. Romdhane, "Prediction of the pose errors produced by joints clearance for a 3-UPU parallel robot," Mechanism and Machine Theory 44(9), 1768-1783 (2009).

5. P. Voglewede and I. Ebert-Uphoff, "Application of workspace generation techniques to determine the unconstrained motion of parallel manipulators," Journal of Mech. Design 126(2), 283-290 (2004).

6. L. Meng, D. Zhang and Z. Li, "Accuracy analysis of parallel manipulators with joint clearance," Journal of Mech. Design 131(1) (2009).

7. J. Zhang and X. Du, "Time-dependent reliability analysis for function generation mechanisms with random joint clearances," Mechanism and Machine Theory 92184-199 (2015).

8. M.D. Pandey and F. Zhang, "System reliability analysis of the robotic manipulator with random joint clearances," Mechanism and Machine Theory 58137-152 (2012).

9. F.A. Lara-Molina, E.H. Koroishi, V. Steffen and L.A. Martins, "Kinematic performance of planar 5R symmetrical parallel mechanism subjected to clearances and uncertainties," Journal of the Brazilian Society of Mechanical Sciences and Engineering 40(4) 189 (2018).

10. S. Venanzi and V. Parenti-Castelli, "A new technique for clearance influence analysis in spatial mechanisms," Journal of Mechanical Design 127(3) 446-455 (2005).

11. N. Binaud, P. Cardou and S. Caro, "The kinematic sensitivity of robotic manipulators to joint clearances," In: ASME Internationl Design Engineering Technical Conferences Computers and Information in Engineering (2010).

12. Xu. Dongtao, "Kinematic reliability and sensitivity analysis of the modified Delta parallel mechanism," International Journal of Advanced Robotic Systems 15(1) (2018).

13. K. Jeong, S. Woo-Jin and K. Beom-Soo, "Stochastic approach to kinematic reliability of open-loop mechanism with dimensional tolerance," Applied Mathematical Modelling 34(5)1225-1237 (2010). 
14. G. Cui, H. Zhang, D. Zhang and F. Xu, "Analysis of the kinematic accuracy reliability of a 3-DOF parallel robot manipulator," International Journal of Advanced Robotic Systems 12(2) (2015).

15. Z. Zhan, X. Zhang, Z. Jian and H. Zhang, "Error modelling and motion reliability analysis of a planar parallel manipulator with multiple uncertainties," Mechanism and Machine Theory 12455-72 (2018).

16. D. Zhang and X. Han, "Kinematic reliability analysis of robotic manipulator," Journal of Mechanical Design 142(4) (2020).

17. L. A. Zadeh, "Fuzzy sets," Information and control 8(3)338-353 (1965).

18. S.-J. Huang and J.S. Lee, "A stable self-organizing fuzzy controller for robotic motion control," IEEE Transactions on Industrial Electronics 47(2)421-428 (2000).

19. L. Wang, T. Chai and L. Zhai, "Neural-network-based terminal sliding-mode control of robotic manipulators including actuator dynamics," IEEE Transactions on Industrial Electronics 56(9)32963304 (2009).

20. F.A. Lara-Molina and K.A. Takano and E.K. Koroishi, "Set-point regulation of a robot manipulator with flexible joints using fuzzy control," In: 2015 12th Latin American Robotics Symposium and 2015 3rd Brazilian Symposium on Robotics (LARS-SBR) (2015) pp. 103-108.

21. R. Storn and K. Price, "Differential evolution-a simple and efficient heuristic for global optimization over continuous spaces," Journal of global optimization 11(4)341-359 (1997).

22. S.V. Shah and S.K. Saha and J.K. Dutt, "Denavit-Hartenberg parameterization of Euler angles," Journal of computational and nonlinear dynamics 7(2) (2012).

23. Geng, Xinyu and Wang, Xiaojun and Wang, Lei and Wang, Ruixing, "Non-probabilistic timedependent kinematic reliability assessment for function generation mechanisms with joint clearances," Mechanism and Machine Theory 104(9)202-221 (2016).

24. Wang, Jinge and Zhang, Junfu and Du, Xiaoping, "Hybrid dimension reduction for mechanism reliability analysis with random joint clearances," Mechanism and Machine Theory 46(10)1396-1410 (2011).

25. Geng, Xinyu and Li, Meng and Liu, Yufei and Zheng, Wei and Zhao, Zhijun, "Non-probabilistic kinematic reliability analysis of planar mechanisms with non-uniform revolute clearance joints," Mechanism and Machine Theory 104(9)413-433 (2019).

\section{A. Reliability using the Monte Carlo Simulation}

The Monte Carlo simulation is used to estimate the reliability as presented in the following algorithm. The procedure consists of estimating the rate between the frequency that the positioning error exceeds the maximum limit and the total number of samples $n_{s}$ considered in the simulation. 
Result: $p_{f}$

Definition of the geometric parameters of the manipulator;

Definition of the maximum limit of the positioning error $e_{\max }$;

$n_{f}=1$;

Sample the uncertain parameters of the clearances to obtain a set of $n_{s}$ random inputs ;

// Defined in Eq. (2)

for $i=1: n_{s}$ do

Compute the positioning error $\delta \mathbf{p}_{t}$ for each random input ; // Eq. (9) for serial manipulator and Eq. (10) for parallel manipulator.

if $\left\|\delta \mathbf{p}_{t}\right\| \geq e_{\max }$ then

$n_{f}=n_{f}+1$

end

end

$p_{f}=\frac{n_{f}}{n_{s}} ;$

// Probability of failure

Algorithm 1: Estimation of the kinematic reliability by using the MCS.

\section{B. Convergence Analysis}

In this appendix is evaluated the number of samples necessary to carry out the Monte Carlo simulation. Figure B shows the convergence results for the maximum translational error $\max \left(|| \delta \mathbf{p}_{t}||\right)$ and orientation error $\max \left(|| \delta \mathbf{p}_{r}||\right)=\max |e|$, respectively.

$$
\max \left(\left\|\delta \mathbf{p}_{t}\right\|\right)=\max \left(\left[\left\|\delta \mathbf{p}_{t}\right\|_{1}\left\|\delta \mathbf{p}_{t}\right\|_{2} \ldots\left\|\delta \mathbf{p}_{t}\right\|_{n_{s}}\right]\right)
$$

It is verified that the solution always converge for $n_{s} \geq 1.5 \times 10^{5}$.

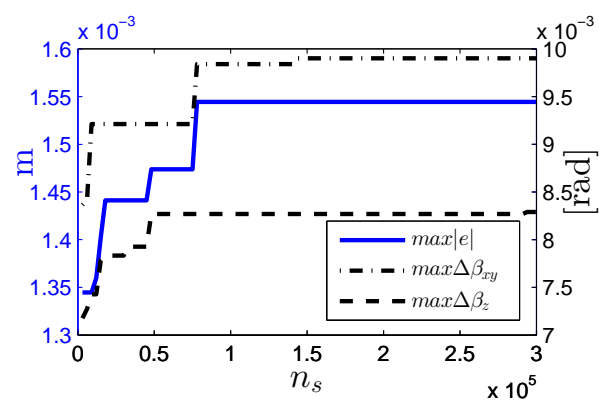

Fig. 16. Convergence in the maximum error for the numbers os samples $\left(n_{s}\right)$ used in the MCS. 